\title{
Efficient Welding Defect Detectionand Classification of Phased Array C-Scan Images Using Modified Fast Fuzzy C Means Andradial Bias Function Neural Network
}

Jayasudha J C ( $\square$ jayasuphd@gmail.com )

Sathyabama Institute of Science and Technology

Lalithakumari S

Sathyabama Institute of Science and Technology

\section{Research Article}

Keywords: 2D AADF, AMA-CLAHE, MFFCM, PMF, GLCM, 2D Band-let Transform, RBFNN

Posted Date: September 28th, 2021

DOl: https://doi.org/10.21203/rs.3.rs-930227/v1

License: (a) (1) This work is licensed under a Creative Commons Attribution 4.0 International License.

Read Full License 


\title{
EFFICIENT WELDING DEFECT DETECTIONAND CLASSIFICATION OF PHASED ARRAY C-SCAN IMAGES USING MODIFIED FAST FUZZY C MEANS ANDRADIAL BIAS FUNCTION NEURAL NETWORK
}

\author{
J.C.Jayasudha, Research Scholar, Faculty of Electronics, Sathyabama Institute of Science and Technology, \\ Chennai, Tamil Nadu 600119. Email: jayasuphd@gmail.com
}

\author{
Dr.Lalithakumari.S, Associate Professor, Department of EIE, Sathyabama Institute of Science and \\ Technology, Chennai, Tamil Nadu 600119. Email: lalithavengat@gmail.com
}

\begin{abstract}
In the recent past, phased array technology is one of the most important methodologies used for inspection of welding. The welding defect identification is a difficult task due to noise content and uneven illumination and contrast on phased array 2D image. Artificial Neural Network (ANN) is a recent Machine Learning (ML) technology that has been achieved a lot of attention over the recent years. The saliency feature extraction for representing image has become complex due to quality of 2D image. The proper image restoration and enhancement techniques should be applied in order to improve the quality of 2D phased array image. The 2D-Adaptive Anisotropic Diffusion Filter (2D AADF) is applied to eliminate noises such as impulse noise and speckle noise. The Adaptive Mean Adjustment-Contrast Limited Adaptive Histogram Equalization (AMA-CLAHE) is the enhancement technique that is applied to improve contrast and brightness of the phased array 2D image. The welding defect region can be exactly segmented using saliency mapping to contour boundaries of defects in welding. In this paper, a novel methodology for welding defect detection is applied based on Modified Fast Fuzzy C Means (MFFCM) clustering technique by integrating Probability Mass Function (PMF) threshold technique for higher range of efficient and accurate segmentation. The Gray Level Co-Occurrence Matrix (GLCM) and 2D Band-let Transform (2D BT) are applied to extract features on segmented image. TheRadial Bias Function Neural Network (RBFNN) classifier is one of the ANN classifier for classifying welding defects. Most of image classification techniques utilize RBFNN as they will provide great range of accuracy and precision while compared to existing techniques. The localized generation error model is implemented in RBFNN in order to minimize Mean Square Error (MSE). The efficiency and accuracy of the proposed methodology has been evaluated with the help of experimental results in terms of graphical representation and numerical analysis.
\end{abstract}

Keywords: 2D AADF, AMA-CLAHE, MFFCM, PMF, GLCM, 2D Band-let Transform, RBFNN

\section{Introduction}

Welding fault represents a major threat to the secure use of oil pipelines. Additionally, there are various shortcomings in machine welding fault identification, like subjectivity and incorrect prediction of design variables. And it is suggested an automated inspection method for welding X-ray images for surface cracks. Basically five faults (holes, absence of penetration, incomplete fusion, round deformities, and stripy deformities) and-anti-defects were selected for identification in the scheme. The system comprises 3 phases: emission of defects, identification of defects, and validation of defects. Context subtraction was implemented in the first step, utilizing an active threshold approach to classify possible defects. In the second phase the adaptive cascade boosting (AdaBoost) methodology was used for 
classification problem to derive real flaws from the huge list of possible faults. Grayscale characteristics and geometric characteristics of the abnormalities for categorization were obtained. In the third phase the multi-classification clustering method was enhanced. A detection accuracy rate is necessary in the process of separating faults from - anti-defects [1].Automated analysis of defects in welding X-ray images is presently a target of much worldwide investigation. Automated computer vision, image processing, and information processing are required in the innovation. It is defined that an image analysis technique for instantaneous detection of defects using digital image fusion that reconstructs a few methodologies such as edge detection, wave shape factor, adaptive threshold differentiation, and divisional weld filtration. Test results indicate that abnormalities that indicate an enormous change over a predetermined magnitude of pixel intensities can be divided up regardless of the number, area, frame, or dimension. The approach is much more reliable and functional than the existing approaches utilizing only one approach [2].Feature extraction plays a key role in automatic detecting defects in welding and in radiographic sample categorization. Among the techniques of differentiation, border removal based on feature extractions is a useful method to characterize the structure, and then conclude the kind of defects under inspection after the analysis stage. A technique for the automatic prediction of machining-defect shapes in radiographic images is defined. The technique provides a mathematical framework of the prediction of the curves by manipulating numerical stress snake based on non-parametric image simulation. Here the boundary energy is supplemented by a province power that is a consequence of region of interest (ROI) statistical properties. Non-destructive testing (NDT) is involved with technique for identifying deformities in particles without in any way modifying the entity. Phased array evaluation is one of the most essential, flexible and commonly recognized methodologies of weld framework evaluation by NDT. Radiographic test includes using designed to penetrate gamma or X-ray to analyze the defective components and structural aspects. Traditionally, expert system verify and define weld X-rays. Additionally, human comprehension of machining radiographs is very contextual, inaccurate, labor-intensive sometimes and incomplete. Consequently, in recent years, various automated inspection techniques for weld radiographs have been tried globally. In factory applications, image processing is a key factor in the successful implementation of quality management within the different mechanisms. Industries using these modern technologies achieve a competitive edge since it leads to greater manufacturing, improving process quality and minimize production costs [3]. The innovative diffusion technique provides abnormalities to be enhanced, sharpened and the context of the image filtered. This current technique is focused on the use of a nonlinear sigmoid function feature to change the traditional diffusion principle. Experimental results are obtained on several actual radiographic historical Gaz piping images from the "Tunisian electric and energy Delivery Community" and the "Management Departments - chemical production research labs: Saybolt-Tunisia" community. Compared to other existing methodologies, the newly designed deficiency detection technique is showing great performance. Industrial x-ray is now an excellently-established procedure for detecting and assessing deficiencies such as divergences, holes, high porosity, destroy thru, and lack of depth discovered in welding process. This technique is based on filtering the image first using an adaptive filter, and then applying an adaptively stretching method to the ROI using a look-up table transform. Second, by implementing texture features, they remove the deficiency which removes tiny holes, gaps, and links the borders tightly [4]. The methodology of Time of Flight Diffraction (TOFD) is commonly used for automated quality control, particularly in the chemical industry, where forging efficiency is important to prevent lost productivity. Even though it offers higher speed evaluation, high sizing efficiency and low level of inaccurate deficiency evidence, the categorization of abnormalities using ultrasonic signal produced by the TOFD methodology is still constantly influenced, as it relies largely on the operator's experience and knowledge. However, the usage of statistical methods for signal pre-processing and pattern identification, such as Artificial Neural Networks (ANN), increases the efficiency of identification of observed defects by this method. During the metalworking process, three types of faults are essential: absence of fusion (AF), absence of penetration (AP) and permeability (PE) were placed into the test samples, producing sequence abnormalities. Using ultrasonic testing and radiographic methods, the role, type and element of each implanted deformity was registered. The Fourier Transform and Wavelet Transform were used to preprocess Ascan signaling which were obtained by 
TOFD methodology throughout inspection of welding. The analysis is able to demonstrate Wavelet Transform's usefulness in pre-processing such types of signals, as the right level in Continuous Wavelet Transform was chosen to include a neural network. In order to differentiate the principal deficiencies in weld metal found by the TOFD method, hierarchical regression classifiers were introduced into the neural network. The findings indicate the successful welding defect detection rate of success in pre - processed TOFD indicators, primarily through the use of Wavelet Transform. In general, the results acquired were very encouraging and might contribute significantly to the development of an automated process for the identification and tracking of investigated welding abnormalities by the TOFD method [5]. A theoretical extension of improved noise-smoothing method to welding X-ray imaging of poor contrast images, using the illustration of laser diffusion capsule experiments of X-ray is applied. Based on the principle of the variation anisotropic differential equations, create a software code that uses discrete powered by noise to optimize the process of de-noising the object. The findings of the measurement reveal an efficient noise filter processing thus keeping the edge profile of noisy data captured with the initial contrast as small as $1 \%$. Solutions of improved de-noising techniques to X-ray objects that help to generate and document phase-contrast trends in the most difficult tests of reduced-absorption intensity segments and sub-objects even without using more complex X-ray machine. Noise reduction filtering can also be helpful for mergers and acquisition of quick X-ray medical imaging or tomography frames, which struggle from a powerful shot-noise background. In contrast, computer object detection can enhance the quality of frequent images for better presentation and analysis [6].Image Histogram Equalization (IHE) based methods are the popular image enhancement methods to improve the contrast intensity of an input image by altering the image pixel rate, based on its allocation of intensity. Using restricted histogram equalization a new contrast image and edge enhancement strategy was developed. A modern mapping method for histogram equalization is developed and a series of restrictions are introduced before the equalization operation on the histogram of an image and then un-sharp masking on the resulting image is implemented. The approach suggested guarantees improved contrast and texture features, which is calculated with respect to differential entropy. Contrast enhancement technique plays an important role in applications for image analysis, such as digital cameras, diagnostic devices, spatial data etc. Histogram Equalization (HE) is among the well-known processes by which an image contrast is improved. By expanding its diverse intensity values using cumulative distribution function, HE allocates the pixel intensity of the input image equally. The big concern with HE is the average change that contributes the average brightness of the input and output images to a significant variance. The proposed methodology achieves three-level edge and contrast adjustment by I) creation of altered HE linear transformation ii) detection of measuring and threshold-based input image histograms and implementation of adapted HE transformation function and iii) non-sharp masking over the resulting image [7]. The goal of enhancing the object is to create a modified image that is more appropriate for particular implementation than the main image. Implementation may include edge identification, threshold detection, image fusion, classification, etc. The various types of temporal domain image enhancement techniques are provided for both the gray scale and RGB color images. For the various algorithms, the quantitative method such as AMBE (average absolute amplitude brightness error), PSNR (Peak signal to noise ratio) and MSE (Average Mean square error) are analyzed. Optimized contrast strength work (OLCSW), non-linear system contrast stretching linear combination (NLLCS), non-linear system contrast expanding acceleration (NLCEA), Bi-Histogram Equalization technique (BHET) methods are applied and evaluated for gray image Weighted gamma correction. For linear contrast stretching of the color image (RGB), Non-linear intensity stretching linear combination and non-linear contrast spreading techniques are applied.During the performance analysis, it was noted that for multiple images certain techniques do offer significantly extremely distinctive features (MSE or AMBE). The innovative enhancement method Local mean and local standard deviation (LMLS) which will begin taking care of issues had been introduced to balance those parameters. Through observational review It was found that the proposed approach provides better values for AMBE (should be low) and PSNR (should be high) compared to other existing algorithms, but these properties are not quite distinctive for various images [8].A great amount of improved Fuzzy c-means (FCM) clustering algorithms have been commonly applied for image segmentation of the gray-scale and color object. 
However, most of them seem to be time intensive and, for 2 purposes, are unable to give required segmented image for color images. The first is that the integration of regional spatial data often creates computational complexity due to frequent attribute - based access control within a regional neighborhood window among grouping facilities and pixel elements. Another is that a normal adjacent window typically splits up the existing local texture image framework and thereby contributes to inadequate differentiation. A fast FCM clustering method (SFFCM) focused on super pixels is proposed which is considerably faster and more reliable than the state-of-the-art proposed techniques for differentiation of color objects. To get better local temporal neighborhoods, initially describe a multi-scale phenotypic gradation reorganization procedure to achieve an overall texture super pixel object. Like typical adjacent windows with fixed size and form, the super pixel image has excellent flexible and abnormal local texture communities that are beneficial to enhance the differentiation with color images.Secondly, the correct color image is effectively standardized obtained super pixel element object, and its histogram is accurately assessed by calculating the number of pixel elements in each area of the super pixel element object. Ultimately, FCM is applied on the super pixel element object with histogram factor to achieve the final segmented image [9].Numerous real-world categorization issues in the feature space are described by measurements of a complex distribution. The classification accuracy is calculated by the exceptional characteristics of all measurements in characteristic sub-spaces. A novel approach is applied for the creation of a subspace learning-based Radial Basis Neural Function Network (RBFNN) classification model. Throughout the learning cycle, the function sub-spaces are acquired per each RBFNN hidden nodes. For various hidden nodes, the weight values among the input layer and the hidden layer are adapted to create various subspaces with dominative characteristics. The entire network and imperious features are encrypted in two sub-populations that are co-evolved together using the co-evolutionary method to obtain a greater global optimization for the RBFNN projections. Usually the feature selection is done to minimize the aspects of the data sets by assessing a subgroup of selected options that is most vital for efficiency in classification. A function selection process, though, typically tests the applicant component subsets over the whole data gathering independent of the evaluation requirements. So, only one applicable set of features, optimal based on predefined requirements, is successfully determined for all measurements belonging to various classes [10].

\section{Related Works}

Du Dong et. al [11] has proposed an automatic assessmentmethodology of metal weld imperfection in radiography X-ray objects. Utilizing enhanced image filtering and image data fusion based probabilistic finest-first search methods, the algorithm detects various types of abnormalities in weld, like slag integration, roll holes, unfinished absorption and fractures and undercut etc. Image preprocessing primarily keeps of two components: image enhancement and image filtering. It recognizes that the non-uniform light readily destroys X-ray images. Optimized histogram equalization method is being used to modify the gray level of the images to modify the visual and to enhance the visual appearance of the images to be filtered.Modification of the intensity is also carried out in this phase. The frequent errors in welding elements are blow hole, grain boundaries, incomplete fusion, lack of depth, melt through and undercut, etc., where deficiencies such as blow hole, grain boundaries, incomplete fusion and lack of depth always occur in the middle of the welding element, all along central axis of the welding element, whereas other deficiencies such as undercut occur primarily near the boundaries of the welding element. And the identification of various surface defects using various techniques is needed. It could even split a weld into many areas, defined as welded zone (ZW) and boundary of Weld (BW), and investigate every other zone for available prior abnormalities using the correlating method.Zhiye Guo et. al [12] has proposed Classification of sensitivity welding position images by convolutional neural network (CNN) dependent process. The characteristics of friction wielding locations are quite varied and complicated, making it more difficult to use difficult limit to differentiate the positive and negative aspects ones. A few

kinds of CNN are constructed to understand the characteristics of welding position, with various levels and layer edges. The training sets are to control images and symbols for the CNN training. Label is an 
image symbol that can signify that the image is in whether the context it resides. There are two phases for preparing a CNN during the training process. The first is propagation forwards. The most essential step, however, is the second that is considered back propagation algorithm. The output resulting from the feedforward propagation will be compared to the actual findings. And this method is termed the role of lost opportunity. The feature indicates how far the expected result is from the real one. And it is usually use reasonable batch curve to learn neural network to alter the error back to the first layer from the last layer. And all network strength should be balanced as per the error. That advance is called modeling.Rafael Vilar et. al [13] has described Automated system welding fault detection scheme in radiography x-ray images. In a first level, image analysis technologies were applied, such noise removal, contrast adjustment, limit and identification, to facilitate in the identification of welded areas and the identification of welding fault processes. In a second level, a set of geometric parameters was implemented and obtained among the fault applicants that define the texture and alignment of the abnormalities. In a third level, an artificial neural network (ANN) for weld fault identification was used for input layer and hidden layer on a project requirements with different implementations. Our objective is to assess this ANN by altering the performance measure in the input and hidden layer for various neurons in order to improve the performance at the identification phase. The measurements of the defect candidates input function vector are broad but the weight vector elements may be strongly clustered and repetitive. Reducing the dimensions of the input function vectors is useful in this case. An appropriate method for carrying out this process is the primary study of the elements. This method has 3 functions: the elements of the input vectors are becoming exactly equivalent (so that they are not highly correlated with one another), it arranges the consequent orthogonal elements (main components) so that those with the biggest variability come initially, and it removes those elements which make a contribution the least to the difference in the set of data.Eric Michel-González et. al [14] has developed Novel hybrid diffusion filter-based de-noising filter design. Instead of using 4 different vectors across the pixel element of interest, it uses geometrical parameters obtained from the dispersion of local pixel element intensity to calculate the diffusion coefficients in vertical and horizontal directions. It is evaluated the filter performance utilizing small dose digital radiography and contrast - Enhanced ct images such as edge preservation histogram equalization and noise removal. In digital x-ray processing, de-noising with edge preservation is very necessary because it will enable one to minimize the dose of x-rays in human subjects without visible degradation of the object.Spatial data knowledge for precise detection is of great importance in de-noising filter architecture for radiography, edge preservation and also noise removal. Quick computing is also necessary in addition to this because digital x-ray objects are largely made up of broad vectors. The suggested de-noising filtering displays comparable results in terms of edge protection and noise removal to that of variation anisotropic diffusion filters (ADFs), on Perona-Malik ADF, as well as the other Weickert's ADF. However, the calculation time has been minimized significantly.Robert J. Burkholder et. al [15] has investigated the coherence factor (CF), Calculated as the fraction of coherent power to incoherent power obtained by a sensing device for a particular moment in an image taken, this ratio is added to the image analysis by walls. CF processing has seen to minimize noise and improve image transparency and homogeneity by eliminating the low-coherence elements. It is also shown that the CF is degraded by cross-wall propagation, allowing its use as a probabilistic metric for auto-focus and image processing methods. The CF is a dimensionless number described as the proportion of the number coherent power the sensing device receives to the maximum incoherent power. The coherence function was introduced to the issue of radar imagery through-wall as a way of measuring the interference induced by transmitting of the wall and enhancing a traditional image through removing low-coherence characteristics that occur as clutter. Quantifying displacement can require the use of auto-focus methods to modify the impact of diffusion through the wall.It also did appear that the CF adjustment makes the image more consistent. The latter structure is due to the low dependency on the CF's dispersing intensity and downstream range. Finally, it should be remembered that the coherence variable is not a replacement for high-resolution reverse imaging and beam-forming techniques, but does in effect complement these strategies in the same way as the back-projection objects were enhanced here.R. Assaf et. al [16] has presented A novel classifier model and online learning paradigm for learning the variables of a RBFNN 
(radial-based neural function network) equalizer in a highly nonlinear time-varying system. The categorization technique is used to calculate the facilities of the nodes in the input layer equitable to the communication effectiveness. This proposed unsupervised learning technique based on the penalized competing protocols in both the K-means and the competition. Its major benefit is neither very much an initializing process nor is needing different numbers of communication effectiveness. The weight links and the distributed of the hidden neurons managed to learn through the gradient descent technique, which relates a new proposed method of training. This style of training incorporates the strengths of both offline and online learning methods, such as flexibility and strong fast convergence. In contrast with the results of the ideal Bayesian compensator as well as those of identical compensator learned by certain established training methods, the results of the RBFNN compensator educated by the suggested approach are demonstrated. All of these performances are achieved using different kinds of streams. This unsupervised method does not allow prior knowledge of the channel state numbers and essentially lowers this amount in two steps. In addition, a new method of training has been proposed that integrates the benefits of both online and offline modes of learning. The suggested methodology and method of training are used for studying the RBFNN compensator variables. Good efficiency is accomplished by different channel forms, and center reduction has been confirmed for highly nonlinear time-varying channels.Daniel S. Yeung et. al [17] has Localized classification error model that bind the generalization error from above inside the training set locality using probabilistic sensitivity calculation. This is then used among defining a generalization error level, to establish a design selection strategy for a classification model with optimum protection of unknown samples. The generalization error limits discovered by error signal designs that use a classifier's set of successful variables and the number of class samples is generally very unstable. Such boundaries are designed for all of the input area. Radial Bias function neural network (RBFNN), Supporting vector machine ( SVM), and multi-layer perception neural network (MLPNN), even so, are local learning systems that are more effective in reducing issues and treating unseen measurements near the training images.Pedro Antonio Gutiérrez et. al [18] has proposed A combined multi-logistic approach, dubbed original and radial base function (RBF) logistic regression. The method to extract the correlations is performed in three phases. First, an adaptive programming (AP) method is implemented to construct an RBF neural network (RBFNN) with less RBF transforms and the simplest and easiest design. Then, the original feature domain (or the response variable area, as widely recognized in logistic regression research) is modified by inserting the variation representations of the response variable provided by the greatest team RBFs in the final generation. Finally, the parameters correlated with an inter-logistic regression model constructed in this expanded variable domain are calculated by a likelihood optimization process. 2 distinct multi-logistic regression methods are implemented in this final stage: one perceives all original and RBF coefficients (inter-logistic early-RBF regression) while the other progressively structures the method and relates classification technique, leading in an optimal classification of variables [simple logistical initial-RBF regression (SLIRBF)]. Both methodologies have included a variable for normalization, which has also been designed. The suggested approach is evaluated using 18 model classification problems arising from the well-known machine learning concerns and two actual agronomic issues. The findings are contrasted with the related multi-logistic regression techniques implemented to the original variable domain, the RBFNNs derived from the EP method, as well as other deterministic classification methods, like numerous RBFNN implementation methods [e.g. relaxing kernel density estimation, support vector machines, a discrete classification (dense multi-nominal logistic regression)] and a protocol. Relative to the related multi-logistic regression approaches and the RBFEP model the SLIRBF systems are considered to be efficient. A statistically significant metric is used which implies that SLIRBF is reaching the state of art.Zheng Qin et. al [19] has presented Particle swarm optimization ( PSO) algorithm for automating RBF network architecture, addressing pattern classification issues. When using a radial-base function neural network (RBFNN) to classify patterns, the scenario requires constructing RBFNN configuration and its facilities and sizes as well. Because of its basic communication networks and rapidly based models, RBF neural systems are increasingly used for performing sequence supervised classification. By using RBF systems to classify patterns, the main challenges are to determine the number of essential things and their center roles and sizes. It is because 
the weights among the secret and the output layers could be determined objectively using the algorithm singular value decomposition (SVD) or other method appropriate for solving linear algebraic expressions until the centers and the sizes have been fixed. RBF system is a combination which has three layers. The input layer keeps of $\mathrm{n}$ groups that describe the input vector components $\mathrm{x}$. The hidden layer consists of certain basic functions which perform mapping function. The stimulation of a hidden neuron is estimated by multiplying the width (generally using the Euclidean requirement) among its middle matrix and the topology produced by the input layer stimulation. In the output layer, the stimulation of a neuron is measured by calculating the weight value of hidden layer output values. Thus, PSO-RBF finds the network capacity and the variables that customize every other neuron: the middle and size of the component of its grounds. Findings in the research have been contrasted with those demonstrating that PSO-RBF may be used to design RBFNN effectively, since it receives channels with high generalization capacity and smaller or comparable size to other approaches.Yutthana Munklang et. al [20] has proposed Segmentation algorithm is one of the key steps in numerous computer vision tasks. Image segmentation is one of the important aspects of texture classification. This categorization is not even an easy issue since for several purposes, e.g. motion, magnitude, and so forth, texture can be non-uniform. A regular application of extracting features is required to aid in this procedure. It is included the Fuzzy C-means (FCM) in the co-occurrence matrix of the gray level (GLCM). In general, use FCM's result to measure eight fuzzy co-occurrence equations for each path. There are four characteristics, i.e. correlation, contrast, homogeneity and energy are calculated from each fuzzy vector of the co-occurrence. It is then evaluate our characteristics on the UIUC, UMD, Kylberg, and the Brodatz data sources using the multi - class support vector machine (yet another-versus-all strategy). It reports the accuracy of the categorization that use the same set of functions derived from the GLCM. For the harvesting of texture features integrate the Fuzzy C-means (FCM) grouping with the GLCM. And in each one of the identities construct eight Blurry Gray Degree co-occurrence levels. Finally, the characteristics of correlation, contrast, homogeneity and energy are computed from each plane. It is using a multi-class support vector machine to show the performance of those features.It is also evaluated the numbers of the categorization with those that use the GLCM measured functions. Eight blurry co-occurrence matrix multiplications are constructed for each position. From every blurry co-occurrence vector measures only comparison, similarity, power and uniformity characteristics. Using the yet another-versus-all technique on the UIUC, UMD, Kylberg, and Brodatz data sets instead check our functionality with the classification problems help vector machine. It compares results of the categorization with the same set of functions derived from the co-occurrence of the gray level. It is found that the features derived from our ambiguous co-occurrence equation give a stronger outcome than those from the standard GLCM description.

\section{Motivation}

Phased array is commonly used as a method of non-destructive testing to evaluate the stability and security of welded procedure. Identification and classification of the weld faults is very necessary to maintain the welding efficiency. A considerable volume of work has been carried out during the past years trying to develop an automated method for the identification and diagnosis of welding processes in constant welding using phased array method. An automated program is proposed to identify welding faults from phased array C-scan images and equate them with the classifiers CNN and RBFNN. RBFNN is proposed as a class identification method for a collection of weld faults in phased array images utilizing appropriate attributes dependent on variation geometric identifiers. Image segmentation and feature extraction play a vital role in automatic detecting defects in welding and in phased array image test categorization. Among the methods of differentiation, boundaries extracting based on feature extractions is a useful method to characterize the structure, and then deduce the kind of defects under examination after the research points. The last step of the reconnaissance scheme is to evaluate the identified / classified deficiencies in the weld based on the acceptance criterion.

\section{Existing Methodology}


The inspection process system will be performed in the existing research utilizing computer vision on the image sequence to enhance the quality of the results. In order to identify the difference of every other weld deficiency sequence, $\mathrm{CNN}$ as one of the image analysis can automatically activate the feature which is appropriate for this issue. Convolutional Neural Network (CNN) categorization involves two stages: image absorption using image regularization, and classification techniques using neural network. Gaussian kernel used for low quality degraded image, it tends to help with image segmentation without losing the main data from the source image, this filter also minimizes interruption or noise presence. The exact welding execution is very often impacted by a complicated welding setting, weld specifications, and human elements. The collected images of welding fracture surfaces usually contain a volume of redundancy material. To efficiently collect the details about the weld fault function for the $\mathrm{CNN}$, the images are subjected for pre-processing. Both layer welds fault images that are pre-processed by sampling, refining, and differentiation. First, to remove the distortion of the original images, the 2D median filter is applied to discard the obtained images of the metal substrate. Second, in order to improve the vertical gradient function of the weld region, the images are improved by gradation analysis. After that, the OTSU algorithm is developed to segregate the weld region deficiency from the other factors in the actual images, which partitioned a suitable region of the weld fracture surfaces to innovative sustainable removal of the attribute.

\section{Disadvantages of existing methodology}

- An undesirable disadvantage of segmentation quad-trees is the rectangular area shape expectation. Regional merging may not portion of the edge detection tree's same division. Since there are both cut-and-merge computing choices available, the begin segmentation may not have to fulfill any of the circumstances of uniformity.

- The efficiency of existing method of region-growing segmentation technique is either oversegmented (too many areas) or under-segmented (too few areas).3. The type of segmentation do not always find shapes spanning numerous disjointed areas.

- The CNN is experiencing high computation time.

- For learning complicated tasks the strong graphics processing unit (GPU) is needed. CNN needs large volumes of data to be qualified for high precision detection. Much of the time, CNN has an over-fitting issue because it's also computationally costly as it needs to use a huge database to train.

\section{Proposed Methodology}

The welding defect detection and classification are proposed using Modified Fast Fuzzy C Means (MFFCM) clustering technique and Radial Bias Function Neural Network (RBFNN) classifier respectively. The suitable image filtering and image enhancement methods have to be used in order to progress the feature of 2D welding phased array C-scan image. The 2D phased array image may contain various noises such as impulse noise, Gaussian noise and speckle noise. The different types of noises are eliminated using adaptive filter. The 2D-Adaptive Anisotropic Diffusion Filter (2D AADF) is used to remove various noises such as impulse noise and speckle noise. The 2D-Adaptive Anisotropic Diffusion Filter (2D AADF) is applied to eliminate noises such as impulse noise and speckle noise. The low quality digital image is converted into high quality digital image by applying Adaptive Mean Adjustment (AMA) is the enhancement technique to improve contrast and brightness of the phased array 2D image. The welding defect area could be properly clustered and threshold in order to detect Region Of Interest (ROI). Modified Fast Fuzzy C Means (MFFCM) clustering technique is applied to group defect region pixel elements on welding image. The Probability Mass Function (PMF) threshold technique is applied on the clustered image to get binary version of segmented image of welding defect region. The Gray Level Co- 
Occurrence Matrix (GLCM) and 2D Band-let Transform (2D BT) are used to extract various features on ROI segmented image. Standard calculations of co-occurrence vectors derive the set of features for the sample classification, but the current approach demands that it be derived from the gradient magnitude occurring from the canny edge detection operator. Co-occurrence equations are a widely used diagnostic method for harvesting of feature vectors, which takes into account relative distances and alignment of pixel elements with co-occurring attributes. The Radial Bias Function Neural Network (RBFNN) classification technique is applied for classifying welding defects. The localized generation error model is implemented in RBFNN toreduce Mean Square Error (MSE).

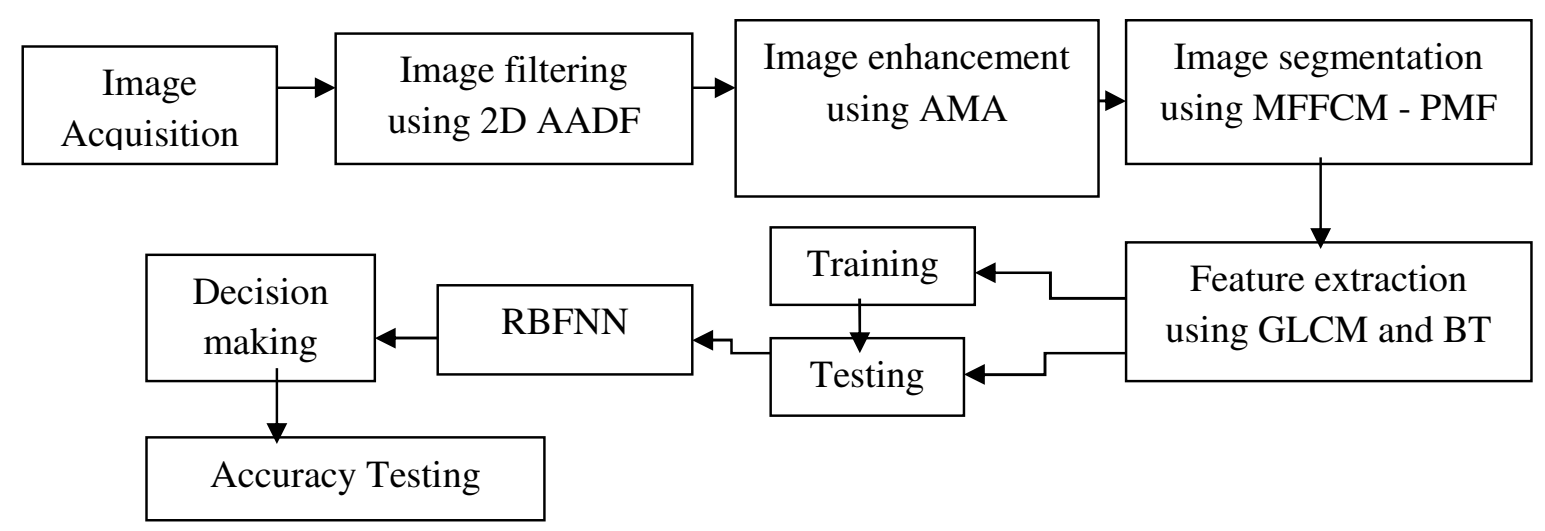

Figure 1 Architecture diagram of proposed methodology

Figure 1 shows the architecture diagram of proposed methodology. In this architecture diagram, the image acquisition is processed in order to acquire phased array welding c-scan images. The image filtering, image enhancement, image clustering, image threshold, image feature extraction and image classification are given.

\section{Phased array image restoration using 2D-Adaptive Anisotropic Diffusion Filter (2D AADF)}

Image filtering with edge preservation in the existence of anomalies is a difficulty in computer vision.2D adaptive anisotropic diffusion filtering is a very well-established technique of edge-preserving digital image filtering. De-noising filters with anisotropic diffusion are not reliable to give impulse noise. In the availability of outliers, they utilize two or higher phases for reliability, and hence require significantly improved mathematical functions for reliability; thus, they are not energy efficient. A small 2D Adaptive Anisotropic Diffusion Filter (2D AADF) image filtering system and introduces an inherently stable and energy efficient technique for anisotropic diffusion filtering of images. The algorithm results the fundamental reliable filtering techniques in terms of the standard efficiency parameters and visual consistency. Image filtering is a fundamental operation in accessing digital images. One wellimplemented solution to image smoothing is filtering of the scale-space. The simple scale-temporal filter is an isotropic filtering diffusion filter that generates image filtering with low conservation of edges. The approach is built on the concept that the near future quality of target pixel strength can be estimated in terms of calculating the pixel element intensity increase or decrease in terms of spatial variables with 
reference to neighboring points inside an available subclass. The free subset consists of four neighboring points in the directions of the north, south, east, and west with reference to the middle pixel element of a window of $3 \times 3$. The prediction equation is a new mathematical formula derived from the probabilistic reason for Brownian motion developed by Einstein. A target pixel element, four neighboring pixel elements, a weighting objective, and a correct data are included in the mathematical formula transforms the statistical concept into an equation. For statistical algorithm simulation, spatial compounds are computed along the target pixel's element north, south, east, and west all directions; an average approximation of the spatial derivatives is acquired. The sum of the target pixel amplitude is modified by applying a value in relation to the lateral derivatives average calculation. Continue this phase for all of the pixels elements in the image. The whole process shall be repeated $n$ cycles until the output of the stated signal to noise ratio is obtained. For evaluating the method different test objects that are distorted with specific noise forms at varying noise levels of intensity are chosen. Diffusion filtering of images in a stable setting, with edge retention, is an increasing research field. Existing reliable diffusion smoothing filters utilize two or three stages, and sorting algorithms are typically complex. This derives from a low mathematics variability framework and a technique for robust image diffusion filtering. In Gaussian, binary, and combined noise scenarios, the modalities good performance in terms of normal performance measures and visual outcomes. In the sense of power intensive applications the inherent mathematical complexity of the proposed system and methodology is relevant.

\section{Phased array image enhancement using Adaptive Mean Adjustment- Contrast Limited Adaptive Histogram Equalization(AMA-CLAHE)}

Effective Contrast enhancement with Adaptive Mean adjustment-Contrast Limited Adaptive Histogram Equalization (AMA-CLAHE) focused on contrast adjustment (CA) is recommended for identification of welding defects. This is recommended that the AMA-CLAHE show the finer obscure features of welding images and change the contrast enhancing rates. The proposed method of welding $\mathrm{X}$-ray images is evaluated. The efficiency of the system proposed is achieved with Peak Signal to Noise Ratio (PSNR). Many common enhancement strategies such as Un-sharp Masking (USM), Histogram Equalization and Contrast Limited Adaptive Histogram Equalization (CLAHE) equate the output. Histogram Equalization allocates the amplitude of the signal homogeneously. But it causes over-enhancement of the source image resulting in the lack of spatial information in the phased array images. AHE varies from the normal histogram equalization from that HE produces only one histogram, because while proposed technique calculates a few histograms, correlating to a separate segment, and uses that to reallocate pixel intensities values. AHE is a better technique of enhancing images. It does however have a noise disadvantage over attenuation. AMA-CLAHE is a type of AHE that minimizes the enhancement of interference. Utilizing CLAHE was identified that it is not so appropriate for very technical aspects for radiographs. The author, together with CLAHE, has implemented global histogram adjustment in AMA-CLAHE. But regional specifics are more essential for the identification of welding deficiency pixel elements in radiographic images than global information. Soa regional contrast enhancement in the suggested protocol to recognize the intricate details hidden throughout the image, and an improvement variable to regulate the improvement rate along with regular CLAHE is used. Thus the incorporation of CA with AMA generates an efficient improvement of contrast with all regional image data that cannot be acquired using regular CLAHE. AMA-CLAHE is a method used to enhance regional image contrast. It is a generalization of the equilibrium of normal histogram and the equalization of the adaptive histogram. AMA-CLAHE does not work like normal Histogram Equalization (HE) over the entire image, but appears to work on isolated regions in images, called tiles. The contrast of every tile is improved, so that the histogram of the output area corresponds rigid to the histogram identified by the variable distribution. Based on the size of input image this variable may be chosen. The neighboring tiles are then mixed using bi-linear interpolation to remove arbitrarily caused borders. The contrast may be reduced, especially in homogeneous areas, to prevent magnifying any unnecessary material, such as noise, that may be accessible in images. To avoid exhaustion the AMA-CLAHE algorithm reduces the slope integrated with the gray level classification 
system. This task is done by permitting in every of the bins linked with the neighborhood histograms only a highest number of pixel elements. After the histogram is "clipped," the clipped pixel elements are transferred equivalently over the entire histogram in aim to maintain the maximum histogram number similar.

The proposed method can be derived into phases to obtain as given below,

1. The phased array c-scan welding image is broken down into continuous and non-overlapping spatial areas. Everyappropriate region size is $\mathrm{M} \times \mathrm{N}$ pixels;

2. The histograms are estimated for everyappropriate area;

3. The histograms are concise for everyappropriate area.

To threshold the peak slope, a clip threshold $\beta$ is used to threshold all the histograms. This is a contrast element that avoids over-saturation of the image especially in uniform environments. Thanks to several pixel elements appearing in the same gray level spectrum these regions are distinguished by a large peak in the histogram of a particular image sheet.

This clip thresholdcan be correlated to clipping factor, $\alpha$ in percent, as given below,

$\mathrm{B}=\mathrm{MN} / \mathrm{L}[1+\alpha / 100(\operatorname{Smax}-1)]$

Where, $\mathrm{M} \times \mathrm{N}$ are quantities of pixel elements of every region and $\mathrm{L}$ is the amount of gray-scale values. When the clip element is equal to zero, then the clip threshold is precisely equal to (MN / L), so if the clip threshold is equal to 100 the maximum permissible gradient is Smax. By still X-ray images Smax is usually set to four. However, by research study it is suggested to get a great option for Smax for any implementation. As the clip aspect changes from zero to hundred the highest slope changes from 1 to Smax for each modeling. Finally, the consequent contrast dependent histograms are calculated by cumulative distribution functions (CDF) for gray-scale modeling. At the four neighboring reference grid pixel elements the outcome modeling at a certain pixel element is normalized from the reference matrices. Special processing of pixel elements in the boundaries of the image beyond the reference pixel elements is necessary. The adjacent tiles were mixed using bi-linear interpolation according to updated histogramthe gray scale measurements were changed.

\section{Welding image segmentation using Modified Fast Fuzzy C Means (MFFCM)}

The fuzzy c-means (FCM) method is among the most frequently used technique for image analysis. Moreover, in this particular case, traditional FCM is not successful by itself, since will be unable to cope with the specific transaction of images, the neighboring pixel elements are strongly linked. By eliminating the precision result in a high vulnerability to vibration and a variety of other objects in image can be done. Previously, many approaches were provided to enhance the efficiency of differentiation. Most require the use of regional spatial data: a pixel element owns gray scale level is not the only data contributing to its assessment task to the selection cluster. The neighbor often exercises their control when they get a name. By adding a spatial cost, the FCM criterion method helps the effective method to approximate temporally consistent membership function parameters. A cluster aggregation additive concept is implemented as an algorithm named Modified Fast Fuzzy C Means Clustering (MFFCM) into objective method of FCM. In level set calculation, this method has its own advantages, but it calculates the neighboring word in any step of evaluation, making the method a significant computation time. In fact, the zero differential situations create a significant number of wrong classifications when calculating the bias expression. Certainly the most pertinent issue of all proposed methods, MFFCM is the reality that they rely on at least 
one variable, the significance of which must be experimentally detected.In the case of the former three, the variable $\sigma$ regulates the neighboring influence, whereas in MFFCM, $\lambda g$ governs the balance among temporal and gray scale components. In MFFCM each pixel element processed gray scale is measured as a weighted average of its adjacent image pixels. Even if it is a dependable, noise-free cost, needing renounced the initial magnitude of the pixel value inevitably generates some additional blur in the image pixels. Precise segmentation involves mitigating this sort of impact.

In the proposed methodology, significant modifications have done on FFCM to improve clustering efficiency without rejecting to clustering based on pixel histogram speeds. In other words, complex formulation has been made that can remove relevant feature information from the image when being implemented as a pre - processing step, such that depending on its histogram, the processed image can be grouped easily afterward. The process suggested is composed of the following steps:

1. A small squares or diamond-shaped cluster Nk surrounding is described. It is due to searching for the focused quality of pixel element $\mathrm{k}$. In this analysis, rectangular windows of size $3 \times 3$ have been used, although certain window types and forms (e.g. diamond) are appropriate as well.

2. Inside the cluster $\mathrm{Nk}$, it is searching for minimum, average, and median pixel level and designates them by mink, maxk, and medk.

3. It is substitute the gray point of the higher and lower pixel element cost with the median cost (if there are more than one peak or low, remove both of them) until they are in the center pixel element $\mathrm{k}$. Pixel $\mathrm{k}$ remains constant in this particular situation, merely branded as an inaccurate attribute.

4. Using the method to measure the mean quadratic gray disparity of the pixel elements in the region $\mathrm{Nk}$,

$\sigma_{k}=\sqrt{\gamma \mid V_{k}\left[y_{r}-y_{k}\right]^{2}}$

5. The filter coefficients can be written as:

$c_{k r}=\left(c_{k} r(s) \cdot \operatorname{Ckr}(g) r \rightarrow N k-\{k\}\right.$

$C k r=1, r=k^{x k}(\max (k), \min (k))$

$C k r=0 r=k^{x k} \in(\max (k), \min (k)$

The fundamental pixel element $k$ may have coefficient value 0 if itssignificancevalue could becalculatedundependable, or else it has originalvalue of coefficient. Other neighbor pixel elements may have coefficients value $C k r \in[0,1]$, depending on itstemporalspace and gray pixel disparitythrough the fundamental pixel element. In case of both conditions, superiordetachmentstandards will drive the coefficient values towards 0 .

\section{Algorithm 1}

Proposed algorithm 1 is summarized as given below;

1. Pre-Processing step: for every pixel element of the welding image, calculatesmoothen gray scale cost, using equations (2), (3), (4), (5).

2. Calculate the image histogram of the pre-processing welding image, get the gray scale values $\mathrm{hl}, \mathrm{l}=1$ ,..., q.

3. Iterate $i$ with suitable gray scale level cost, varying through each one. 
4. Estimate new $u$ ilfuzzy cost of membership, $i=1 \mathrm{~K} c, l=1 \mathrm{~K} q$, and then new visamplestandards for the groups, $i=1 \mathrm{~K} c$, using Eq. (4).

5. If there is applicablemodify in the $v i$ costs, go back to step 4. This is verified by evaluated any standard of the dissimilarityamong the original and the previousmatrix vector $\mathbf{v}$ with a fixedtinyconstant $\epsilon$.

The methodology converges speedy. However, the quantity of essentialepochs depends on $\varepsilon$ and on the primary costs of $v i$.

\section{Image threshold using Probability Mass Function (PMF)}

In particular it first allocates a random starting threshold level. The next stage classifies per pixel element into the nearest class. Using Gaussian distribution the mean values $\mu 1$ and $\mu 2$ within each class are expected in the third step. The second and third steps shall be conducted in accordance until slight is the "change" among the step. There are two major concerns to be described here for obtaining Probability Mass Function (PMF):

(i) The equation for the mean $\mu 1$ and $\mu 2$ attributes is particular to the Gaussian distribution. Unless the distribution of the data is not Gaussian otherwise the $\mu 1$ and $\mu 2$ calculations are incorrect.

(ii) The threshold calculation is not reliable, because the calculation is autonomous of the distribution process.

The Gaussian distribution may a symmetric form only. If an asymmetric feature vector is formulated asymmetric feature vector, that technique may not assure proper segmentation. To provide a better precise segmentation, use of a more transmission is required. Gamma function has more forms than random variable, which may be linear which non-symmetric. Therefore, in the absence of non-symmetric pixels, the usage of the random variable can provide more precision in estimating the measures and limits. After choosing that a class $\mathrm{C}$ is not diverse, then and use an early threshold that has to divide it into two subclasses. Deciding the original threshold to initiate the process relies on the image (class) element. If there is evidence to assume that the subject and target share similar areas inside the image, the mean gray level of the image is a reasonable primary value for T. When artifacts are low relative to the region filled by the background (or inversely), so the pixel elements would be controlled by one category of pixels and the mean gray scale is not a primary option as strong. For instances like this a value somewhere between the average and minimal gray levels is a more acceptable initial value for $\mathrm{T}$. It will prefer to use the second form, as in both situations it typically works. The form variable (L) within an image is stable.

\section{Feature extraction using Gray Level Co-Occurrence Matrix (GLCM) and 2D Band-let Transform (2D BT)}

The welding image texture features assumed as the parameter estimates for creating co-occurrence coefficients. The welding image of color is incorporated to the image of gray-scale, again and will receive the matrix of welding image co-occurrence. An image material is defined using five attributes such as energy, correlation and contrast entropy stationary locale.

\section{Steps involved in GLCM algorithm}

Step1 Welding image is quantized

Step2 GLCM matrix is created.

Step3 Feature selection is created

Step4 Test sample variable's' in the experimental resulting fundamentalcharacteristic is restored by the cost of this estimated feature. 


\section{GLCM Description}

1. Execute quantization on welding image data.

The welding image is tested and converted as a single pixel element, and amplitude is measured as a cost for that pixel element.

2. GLCM matrix is created as given below:

$$
P(i, j)=\sum_{x=1}^{N} \sum_{y=1}^{N}\{1,0\}
$$

Where, 1 if $(x, y)=i$ and $I(x+\Delta x, y+\Delta y)=j, 0$ otherwise

This vector is a square matrix of dimension $\mathrm{N} \times \mathrm{N}$ where $\mathrm{N}$ is the quantity of gray scale levels precisethroughout step 1 . The zero offset $\Delta \mathrm{x}, \Delta \mathrm{y}$ providesspaceamong pixel element of attention and its neighbor pixel element. The creation of GLCM matrix is completed as given below:

a. The 's' could be the test sample that wants to be reflected on for estimation.

b. 'W' represents the test sample set sustainingtest sample 's'that is createdbased on the dimension window size.

c. By only defining the test samples of set $\mathrm{W}$, every pixel element $\mathrm{i}, \mathrm{j}$ of the matrix of GLCM is considered as the calculation that two test samples of pixel elements magnitudes $i$ and $j$ arise in a window size. The average of all $\mathrm{i}, \mathrm{j}$ in GLCM matrixcould be the quantity of time period the particulartemporalassociationarise in $\mathrm{W}$.

d. Estimate symmetric vector of GLCM matrix.

i. Estimate transpose of GLCM matrix.

ii. Include the reproduction of GLCM matrix to itself.

e. Regularize the costs of GLCM matrix calculated by separatingevery pixel $i, j$ with the average of all pixels in the GLCM matrix with value to $\mathrm{W}$.

3. Estimate the chosen feature.

This estimationutilizes the costs in the GLCM matrix as given below - contrast, energy, entropy, homogeneity, correlation, etc.

4. The test sample $\mathrm{s}$ in experimental resulting characteristic is moved by the cost of the estimated feature.

\section{D-Band-let Transform (2D-BT) feature extraction}

A new form of extracting features is implemented, with extended band-let variables suited to the structure of the image. A band-let base is built from a structural vector stream, which shows the specific patterns along which the gray image rates periodically differ. In implementations, this spatial flow must be designed to create band-let bases that take full benefits of the image spatial frequency. Rather than defining the functionality of the image by edges, which are most frequently described is the functionality of the image using a mathematical matrix flow. Such variables provide frequent changes to the local positions in which the image is. Ortho-normal band-let supports are created by separating image help in parallel geometric stream conditions. Such variables provide frequent changes to the local positions in which the image is. Ortho-normal band-let supports are created by separating image help in parallel spatial flow regions.

$$
\gamma B=\frac{1}{M N} \sum_{g=0}^{\infty} h(g) \min (g), 1-\mu_{L}(g)
$$


In which $\mathrm{M}, \mathrm{N}$ is the $2 \mathrm{D}$ image shape, $\mathrm{h}(\mathrm{g})$ is the gray level value, and $\mu(\mathrm{g})$ is the band-let coefficient $\mathrm{g}$ affiliate cost. The role of ultra-fuzziness quantity is used as limit. Let $\mathrm{T}$ denote the limit value. Even so, the band-let variables whose descriptive statistics exceed $\mathrm{T}$ will stay, and the residual correlations will be set to 0. For all band-let variables in the estimation range, the above method for limit is replicated independently again. And a limit in the estimation level for band-let factors is calculated using the position may have the highest estimate of hyper-fuzziness and the value is rendered dependent on this level. Instead a limit is reached again dependent on the optimization of the hyper-fuzziness calculation and test is done for the residual factors.

\section{Phased array image welding defect classification using Radial Bias Function Neural Network (RBFNN)}

Radial base function networks often feed forward and only have one hidden layer. Along with its quicker learning ability as comparison to other feed forward neural networks, a RBFNN is considered a better choice for achieve higher levels. For typical RBFNNs, respectively, the logarithmic rule and the least Squares (LS) criteria are chosen as network input layer and optimization method. A network adjusts the variables of every node incrementally by reducing the LS requirement in accordance with the gradient descent technique. Because a neural network can perform a highly nonlinear modeling from feature space to output space, all developed systematic may be interpolated with the estimated curve created by the neural network. Like a more frequently used neural networks, RBFNNs comprise three layers of modules but with Gaussian or irregular kernels forming the middle (hidden) layer. An amount of kernels are placed in the input space using one of several feasible methodologies for the positioning. Like in neural network, the system inputs are modules that essentially transfer each of the control signals to the centers of the inner layer. The architecture keeps of single hidden layer and single output. This sub-surface design has a significant advantage over hidden layers layer nets in aspects of simulating speed.

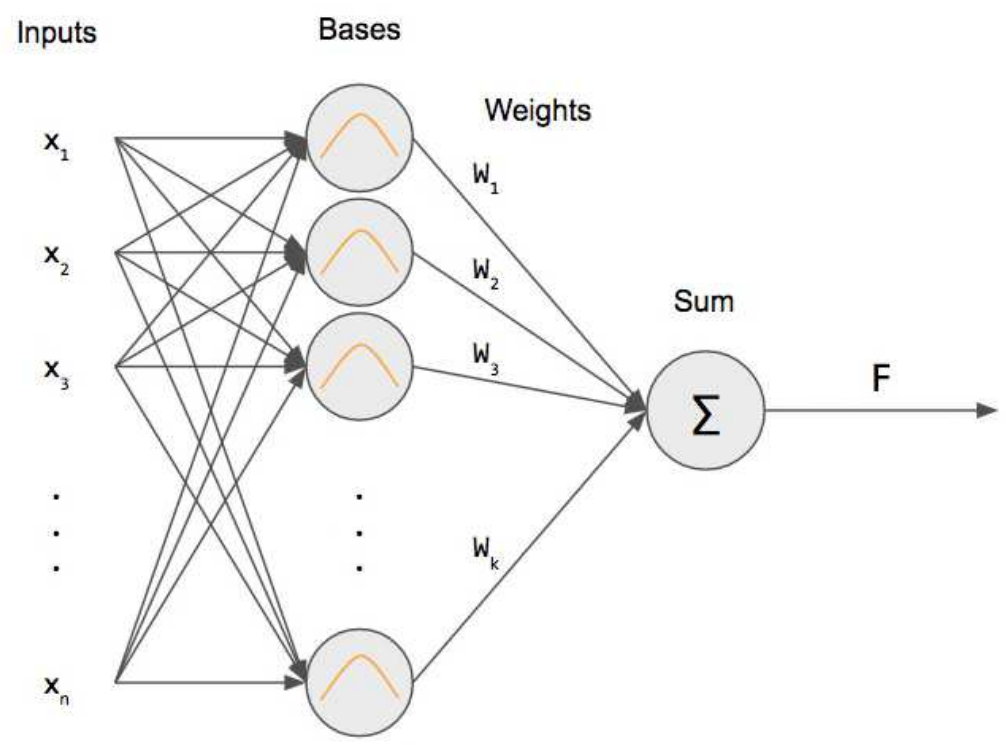

Figure 2: Architecture diagram of RBFNN classification

\section{Algorithm 2}

\section{Algorithm for RBFNN}

Step 1: Place the quantity of population, measurement(number ofhidden layers), Search tendency and executioncircumstance (quantity of iterations). 
Step 2: Place the lower and upper limits of weight vector Wij as(Wmin , Wmax), the lower and upper limits of width Wij as (Wmin , Wmax), the lower and upper limits ofcentre Ci as (Cmin , Cmax), and primary weight Wij, width Wi , centre Wi to effort as trees structure.

Step 3: The position (principles) createdfor the trees like weight Wij, width $\mathrm{Wi}$, centre Ciis calculatedbased on the fitness function $\mathrm{f}(\mathrm{a})$ to confirm its union.

Step 4: Produce new kernelposition(principles) for the trees like weight Wij, width Wi centre Ci using the selected rij and search tendency,and assess the fitness and restorethe trees if the kernelcreated optimized fitness thantrees.

Step 5: Start up for the executioncircumstance (quantity ofiterations is located as 100 in this issue)

Step 6: If the executionsituation is met, continue to thesubsequently step, else producenovel tree position and kernelposition and compute the character fitness from step2, step 3 and step 4.

Step 7: Allocate the optimized costs of weight Wij, width Wi,centre Ci to the RBFNN and accumulate the experimental result.

RBFNN structure is given as seen in figure 2. It will recognize score q. The information is grouped in the input layer. Then, it calculates the middle and the size of every group. The input layer transfers the $d$ signals to the secret layer of $m$ neurons. Each hidden neuron is linked to the center and size of each group which defines an RBF of the input parameters. K-means grouping method determines the center and the width variables of RBF. The quantity of neurons hidden equals the number of clusters. The weights among the secret layer and the output layer can be determined computationally using the process Minimum Least Square (MLS) as in the regression analysis. The MLS gets weights which reduce the Average Square Error (ASE). To construct the RBFNN model has to describe the architecture's inputs and outputs. The inputs are represented as the lung image variables produced by abstraction of the Gray Level Co-occurrence Matrix (GLCM). The GLCM approach is commonly used to generate image parameters for several issues with the identification. GLCM's achieved variables can help to understand the image information in terms of shape. 12 variables as considered as inputs; these are entropy, contrast, energy, similarity, sum of entropy, variance, moment of inverse difference (IDM), mean sum, variance of sums, and entropy of variation, maximum probability and uniformity.

\section{Convolutional Neural Network (CNN) classification technique}

In this research, welding defect classification is done using RBFNN. The Convolutional Neural Network $(\mathrm{CNN})$ is used to compare with RBFNN. The CNN is the one of the types of Deep Learning (DL) technique for classification. In this investigation the input welding image uses the initial image without proceeding through the imaging process. Separation of features utilizing convolution mechanism and gradient descent genetic algorithm for classification tasks, linear interpolation comprises of many separate filers with each executing its own convolution and then scaling them on edge of each other to construct a 3-dimensional feature vector where complexity is defined by number of filters. Categorization using CNN does not need to scan properties and identify welding fault features separately, but the functionality is immediately extracted via the method of matrices. 


\section{Experimental setup and methodology}

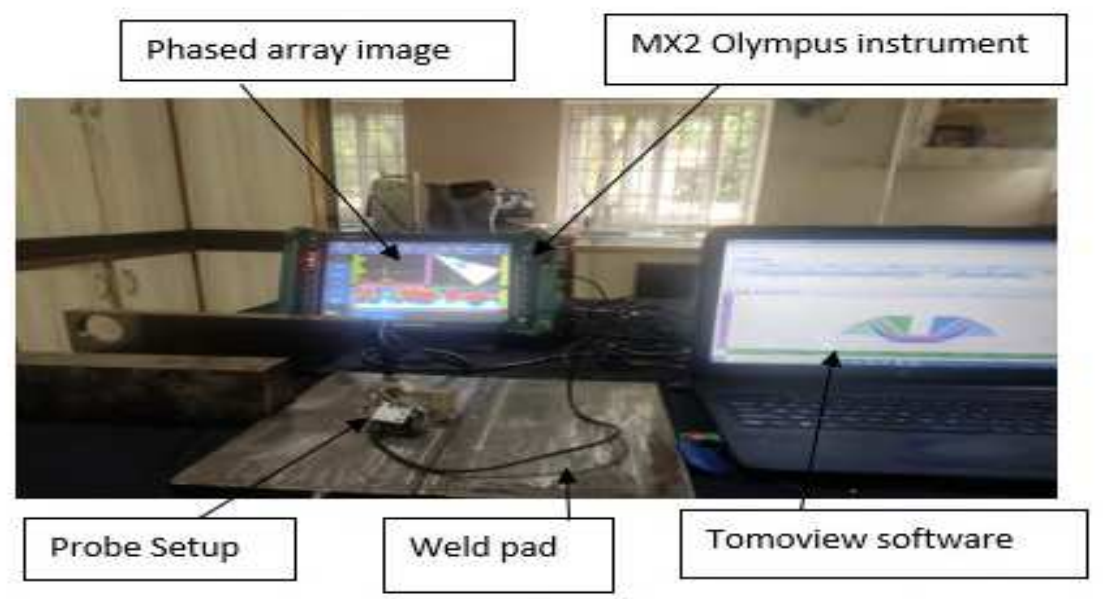

Figure 3: Experimental bench setup of measurement

Figure 3 represents the experimental set up consisting of a weld pad, probe set-up, Omniscan MX2 olympus device to obtain the phased array scan images and which inturn is connected to laptop with the tomoview software to acquire the image, so that further processing and analysis can be done.Inspection of two welded plates with ID number PL16935 and PL16936 as shown in figure (4) was done using Phased ArrayUltrasonic Testing System Omniscan MX2 equipment with 5L64-A12 probe attached to SA12-N55S wedge is used in the experimental setup. Theequipment was neatly calibrated on a V1 block. The couplant used for this testing purpose was glycerin. An encoder was also attached tothe probe to track the distance of probe travel. The encoder was a 12 steps/mm quadrature type. Data was recorded in C-Scan presentation. The result of inspection was then analysed and all discontinuities were measured using the proposed algorithm.

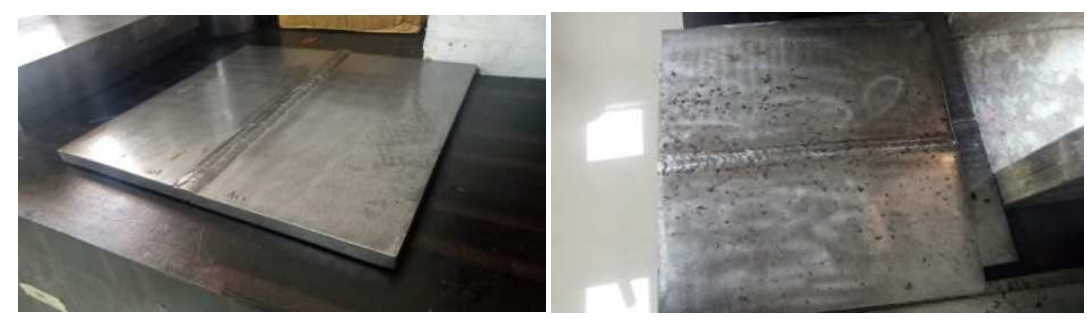

Figure 4: Welded plate images of PL16935 and PL16936

\section{Results and discussion}

This section focuses on experimental discussion on segmentation in phased array C-scan images. A significant factor in welding defect segmentation is noise. Thus, this section includes various sub-sections such as: pre-processing including image filtering and image enhancement, image segmentation, feature extraction and classification. In the preprocessing, 2D-Adaptive Anisotropic Diffusion Filter (2D AADF) is implemented. The proposed filter is capable of eliminating various noises such as impulse noise, speckle noise and Gaussian noise. The figure 5 (a) shows the input raw image of phased array welding image. The filtered image is shown in the figure 5 (b). The contrast and brightness of filtered image is 
improved by applying Adaptive Mean Adjustment-Contrast Limited Adaptive Histogram Equalization (AMA-CLAHE). The enhanced image is shown in figure 5 (c). The image segmentation is one of the commonly used methods for the automatic defect segmentation of c-scan images. The critical part of this method is background modeling. Compared with the welding image, the defect is much smaller. Therefore, when building the background image, the surrounding pixels are considered. The Modified Fast Fuzzy C Means (MFFCM) algorithm is applied for clustering of defective pixels on welding image. As shown in figure 5 (d), the defect region is darker and smaller than its surrounding pixels. The Probability Mass Function (PMF) threshold technique is applied to extract Region of Interest (ROI) in welding image. The figure 5 (e) shows threshold image of welding defect. The figure 5 (f) shows segmented image of welding defect using MFFCM-PMF. All the experimental outputs of figure 5denote welding defect namely slag identification using Phased array ultrasonic Testing (PAUT). Various experimental results of Figure 6 denotes the welding non-defect detection.
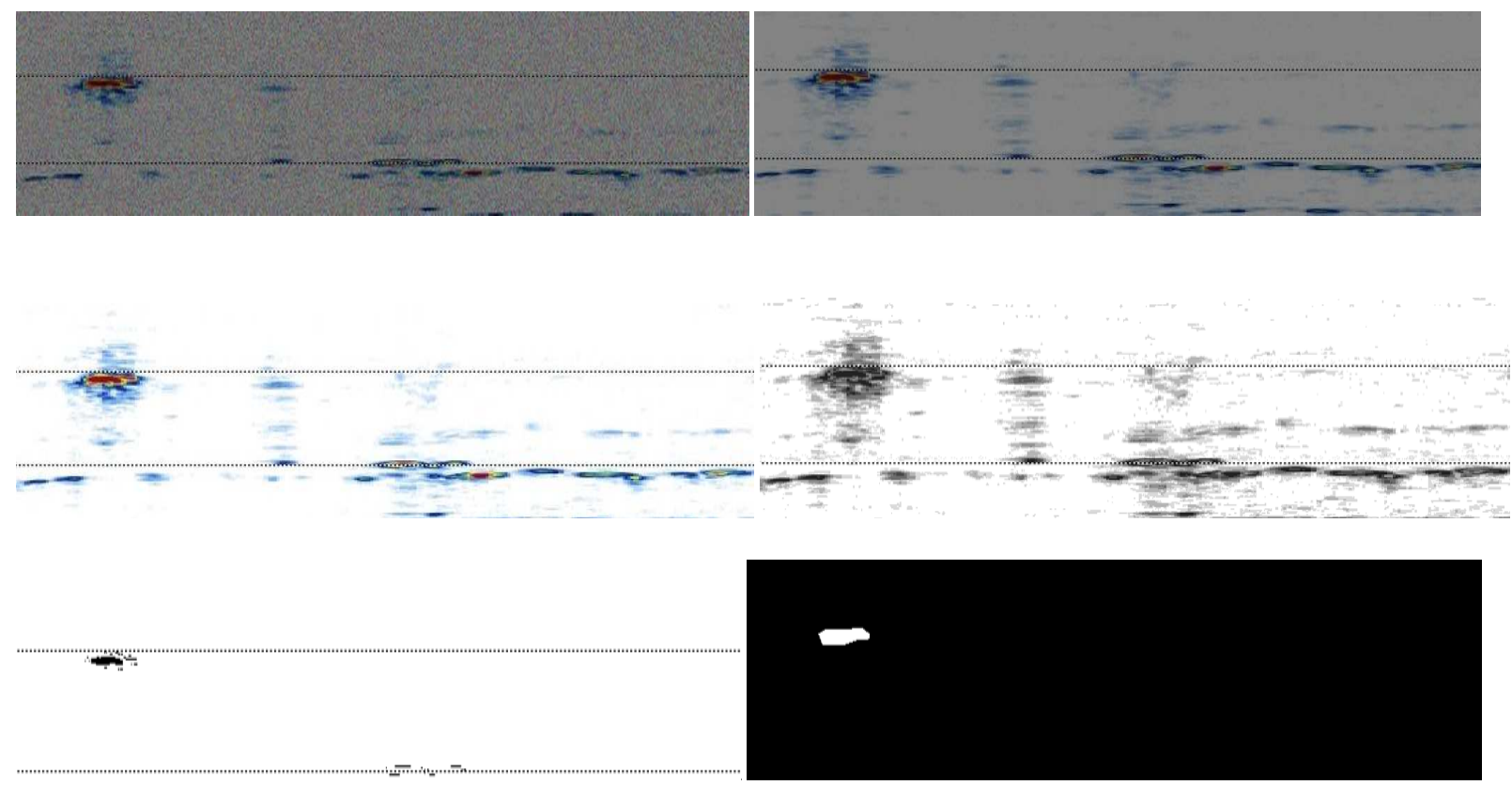

Figure 5: (a) Input phased array welding image with defect noisy image, (b) Filtered image using 2D AADF, (c) Enhanced image using AMA, (d) Clustered image using MFFCM, (e) Threshold image using PMF, (f) Segmented image using MFFCM- PMF.
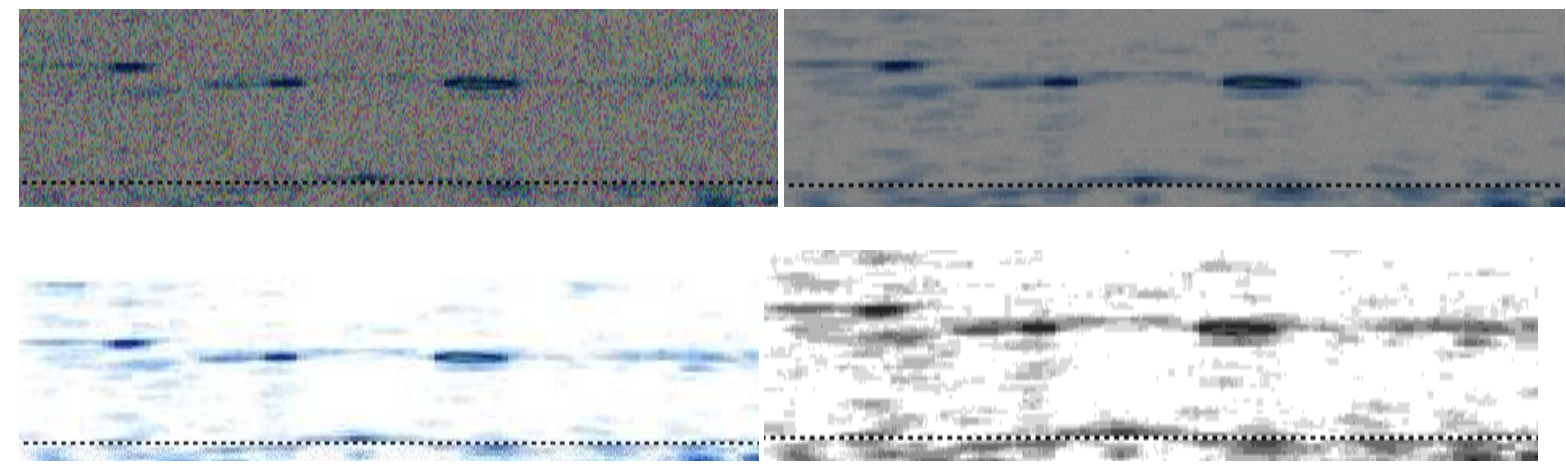
Figure 6(a) Input phased array welding image without defect noisy image, (b) Filtered image using 2D AADF, (c) Enhanced image using AMA, (d) Clustered image using MFFCM, (e) Threshold image using PMF, (f) Segmented image using MFFCM- PMF.
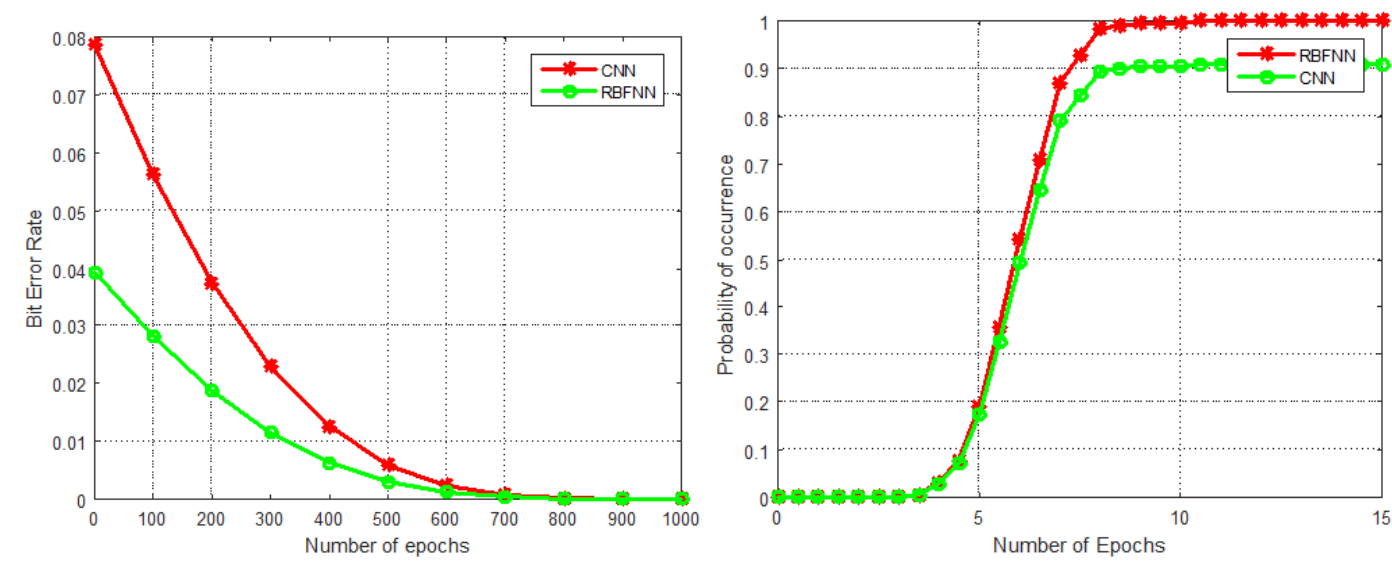

Figure (7) (a) Graph of BER Vs Number of Epochs, (b) Probability of occurrence Vs Number of Epochs

Figure 7 (a) shows the graphical representation of Bit Error Rate (BER) reduction with respect to number of epochs. The CNN and RBFNN are compared in the graph. The RBFNN performance is better than $\mathrm{CNN}$ as shown in the figure. Also, the probability of occurrence of RBFNN is better than CNN as shown in figure 7 (b).

TABLE 1 The Summarized information of welding image feature extraction

\begin{tabular}{|l|l|l|l|}
\hline S. No & Parameters & $\begin{array}{l}\text { Defective } \\
\text { Welding image }\end{array}$ & $\begin{array}{l}\text { Non-Defective } \\
\text { Welding image }\end{array}$ \\
\hline 1 & Entropy & 0.9 & 0 \\
\hline 2 & Energy & 0.5 & 0 \\
\hline 3 & Contrast & 0.6 & 0 \\
\hline 4 & Similarity & 0.8 & 1 \\
\hline 5 & Sum of entropy & 0.7 & 1 \\
\hline 6 & variance & 1.2 & 1 \\
\hline 7 & IDM & 2.5 & 1 \\
\hline 8 & mean sum & 1 & 1 \\
\hline 9 & variance of sums & 1 & 0 \\
\hline 10 & entropy of variation & 0.8 & 0 \\
\hline 11 & maximum probability & 1 & 0 \\
\hline 12 & uniformity & 1 & 0 \\
\hline
\end{tabular}


Table 2 Accuracy Testing using Confusion Matrix (CM)

\begin{tabular}{lll}
\hline $\begin{array}{l}\text { Testing } \\
\text { formula }\end{array}$ & Description & CM \\
\hline
\end{tabular}

1. Accuracy (A) Accuracy conclude the accuracy of the proposed algorithm in forecasting occurrence.

2. Precision $(\mathrm{P})$ Classifier precision/accuracy is calculated by Precision measurement

$$
\begin{aligned}
& \mathrm{A}=(\mathrm{TP}+\mathrm{TN}) /(\text { Total } \\
& \text { no of samples })
\end{aligned}
$$

$$
\mathrm{P}=\mathrm{TP} /(\mathrm{TP}+\mathrm{FP})
$$

\section{Table 3 Performance analysis using CM}

\begin{tabular}{llcc}
\hline Classification & Algorithms & Precision in \% & Accuracy in \% \\
\hline ANN & RBFNN & 99 & 98 \\
DL & CNN & 87 & 84 \\
\hline
\end{tabular}

\section{Conclusion}

The cost of welding defects like gas leakage and oil leakage comprise a key risk to the protected process of pipelines. In this research, efficient welding defect detection and classification system is proposed. The massive non-destructive testing is carried on using various types of image processing techniques. The preprocessing technique is carried out to improve the quality of the phased array C-scan image. The 2DAADF is proposed to filter noisy phased array image. The AMA-CLAHE algorithm is applied to improve contrast and brightness of the image. MFFCM clustering technique is proposed to group welding defect region on the image. The PMF threshold technique is used to extract region of interest. The feature extraction is done for further classification. The RBFNN and CNN classification techniques are used for classification of defect namely slag. Based on the confusion matrix, the performance of both classification techniques is compared. The experimental and graphical representation proves that RBFNN classification is better than CNN.

\section{Declaration:}

Ethics Approval and Consent to Participate:

No participation of humans takes place in this implementation process

Human and Animal Rights:

No violation of Human and Animal Rights is involved. 
Funding: No funding is involved in this work.

Conflict of Interest: Conflict of Interest is not applicable in this work.

Authorship contributions:

There is no authorship contribution

Acknowledgment :

No Acknoweldgements

\section{References}

[1]. Feng Duan, Shifan Yin, Peipei Song, Wenkai Zhang, Chi Zhu and Hiroshi Yokoi, "Automatic Welding Defect Detection of X-ray Images by Using Cascade AdaBoost with Penalty Term", DOI:10.1109/ACCESS.2017.

[2]. Tian Yuan, Du Dong, Cai Guorui, Wang Li , Zhang Hua, “Automatic Defect Detection in X-Ray Images Using Image Data Fusion", 2006.

[3]. Aicha Baya Goumeidane, Mohammed Khamadja and Nafaa Naceredine, "Bayesian Pressure Snake for Weld Defect Detection”, Springer 2009.

[4]. Issam Ben Mhamed, Sabeur Abid and Farhat Fnaiech, "Weld defect detection using a modified anisotropic diffusion model", Springer 2012.

[5]. Elineudo P. Moura, Romeu R. Silva, Marcio H. S. Siqueira and Joao Marcos A. Rebello, "Pattern Recognition of Weld Defects in Preprocessed TOFD Signals Using Linear Classifiers", DOI: 10.1007/s10921-004-0822-4.

[6]. I.A.Artyukovand N.N.Irtuganov,"Noise-Driven Anisotropic Diffusion Filtering For X-Ray Low Contrast Imaging", DOI 10.1007/s10946-019-09782-8.

[7]. P. Shanmugavadivu1 and K. Balasubramanian, "Image Edge and Contrast Enhancement Using Unsharp Masking and Constrained Histogram Equalization”, Springer 2011.

[8]. Shivaprasad Clitus Neil Dsouza, "Edge Preserving Image Enhancement For Color And Gray Scale Images Using Local Mean And Local Standard Deviation “, IEEE 2017.

[9]. Tao Lei, Xiaohong Jia, Yanning Zhan, Shigang Liu, Hongying Meng and Asoke K. Nandi, "Superpixel-based Fast Fuzzy C-Means Clustering for Color Image Segmentation”, IEEE 2018.

[10]. Jin Tian, Minqiang Li, Fuzan Chen and Nan Feng, "Learning Subspace-Based RBFNN Using Coevolutionary Algorithm for Complex Classification Tasks", IEEE 2016.

[11]. Du Dong, Cai Guo-rui, Tian Yuan, Hou Run-shi and Wang Li, "Automatic Inspection of Weld Defects with X-Ray Real-Time Imaging”, Springer 2007.

[12]. Zhiye Guo, Shaofeng Ye, Yiju Wang and Chun Lin, "Resistance Welding Spot Defect Detection with Convolutional Neural Networks", Springer 2017, DOI: 10.1007/978-3-319-68345-4_15.

[13]. Rafael Vilar, Juan Zapata and Ramon Ruiz, "Classification of Welding Defects in Radiographic Images Using an ANN with Modified Performance Function", Springer 2009.

[14]. Eric Michel-Gonzalez, Min Hyoung Cho and Soo Yeol Lee, "Geometric nonlinear diffusion filter and its application to X-ray imaging", 2011.

[15]. Robert J. Burkholder and Kenneth E. Browne,"Coherence Factor Enhancement of Through-Wall Radar Images", IEEE 2010. 
[16]. R. Assaf, S. E. Assad,Y. Harkouss M. Zoaeter, "Efficient classification algorithm and a new training mode for the adaptive radial basis function neural network equalizer",DOI: 10.1049/iet-com.2010.0814.

[17]. Daniel S. Yeung, Wing W. Y. Ng, Defeng Wang, Eric C. C. Tsangand Xi-Zhao Wang, "Localized Generalization Error Model and Its Application to Architecture Selection for Radial Basis Function Neural Network", IEEE2007.

[18]. Pedro Antonio Gutierrez, Cesar Hervas-Martinez and Francisco J. Martinez-Estudillo, "Logistic Regression by Means of Evolutionary Radial Basis Function Neural Networks",IEEE 2011.

[19]. Zheng Qin, Junying Chen, Yu Liu and Jiang Lu,"Evolving RBF Neural Networks for Pattern Classification",2007.

[20]. Yutthana Munklang, Sansanee Auephanwiriyakul and Nipon Theera-Umpon,“A Novel Fuzzy Cooccurrence Matrix for Texture Feature Extraction”, Springer 2013. 